\title{
Diverse Promotive Action of Pyridinecarboxylic Acids on Flowering in Ornamentals and Seedling Growth in Vegetable Crops
}

\author{
Shigeru Satoh
}

Additional information is available at the end of the chapter

http://dx.doi.org/10.5772/intechopen.75636

\begin{abstract}
This chapter describes our recent findings on diverse biological effects of pyridinecarboxylic acids, both pyridine- $d i$-carboxylic acids (PDCAs) and pyridine-mono-carboxylic acids (PCAs), on plant growth processes. PDCA analogs promoted flowering and extended display time (vase life) of cut flowers of spray-type carnation. 2,3-PDCA and 2,4-PDCA were most active in the promotion. Apart from these actions, some of PDCAs and PCAs stimulated root and shoot growth of lettuce, carrot, and rice seedlings. Studies on structureactivity relationship of the chemicals showed that one of the most effective chemicals was pyridine-3-carboxylic acid. Pyridine-3-carboxylic acid is known as vitamin B3 (niacin) and safe for human and animals. These findings suggested the possibility to develop PDCAs and PCAs as novel flower-care agents as well as growth-promoting agents which will be used for vegetable cultivation.
\end{abstract}

Keywords: pyridine-di-carboxylic acids, pyridine-mono-carboxylic acids, pyridine-3-carboxylic acid, flowering, seedling growth, promotion

\section{Introduction}

Pyridine-di-carboxylic acid (PDCA) has six structural analogs, that is, 2,3-, 2,4-, 2,5-, 2,6-, 3,4-, and 3,5-PDCA. In the past, there were a few works on biological activities of PDCA analogs. 2,6PDCA was reported to inactivate markedly aconitase, but 2,3-, 2,4-, and 2,5-PDCA did not [1]. 2,4-PDCA is a structural analog of 2-oxoglutarate (OxoGA) and was shown to inhibit OxoGAdependent dioxygenases by competing with OxoGA [2, 3]. For example, OxoGA-dependent dioxygenases include proline-4-hydroxylase [2-4] and enzymes involved in gibberellin (GA) 
biosynthesis and metabolism, such as gibberellin $3 \beta$-dioxygenase (gibberellin $3 \beta$-hydroxylase), gibberellin-44 dioxygenase, and gibberellin $2 \beta$-dioxygenase (gibberellin $2 \beta$-hydroxylase) [5-9].

Iturriagagoittia-Bueno et al. [10] reported that OxoGA competitively inhibited the activity of 1-aminocyclopropane-1-carboxylate (ACC) oxidase with respect to ascorbate, which was extracted from ripe pear fruits. ACC oxidase catalyzes the last-step reaction of ethylene biosynthesis in plants. Vlad et al. [4] demonstrated that 2,4-PDCA inhibited ethylene production in detached carnation flowers and delayed senescence of the flowers and suggested that 2,4-PDCA inhibited the activity of ACC oxidase by acting as a structural analog of ascorbate. Then, Fragkostefanakis et al. [11] showed that 2,4-PDCA inhibited the in vitro activity of ACC oxidase prepared from tomato pericarp tissues. These results confirmed that 2,4-PDCA inhibits ACC oxidase by competing with ascorbate.

Recently, we examined 2,4-PDCA action on ethylene production and senescence in spray-type carnation flowers to know whether it can be used as a flower-care agent to prolong the display time of the flowers, which had not been referred in the previous paper [4]. Interestingly, we could demonstrate the acceleration of flower opening by 2,4-PDCA in cut spray-type carnation flowers, in addition to retardation of their senescence, resulting in prolonging their display time. We furthermore compared 2,4-PDCA action with those of its structural analogs, such as 2,3PDCA, 2,5-PDCA, and so on, in order to obtain further information on 2,4-PDCA's action. On the other hand, in the course of the above-mentioned study, we found that 2,3-PDCA promoted root elongation, whereas 2,4-PDCA inhibited it in lettuce, carrot, and rice seedlings. We explored the promoting activities of 2,3-PDCA to other PDCA analogs and pyridine-mono-carboxylic acid (PCA) analogs. Also, we carried out a preliminary investigation on the possible biochemical and molecular mechanism of PDCA, mainly with 2,4-PDCA. This chapter describes the details of the effects of 2,4-PDCA and related chemicals on flower opening and display time in carnation flowers, as well as the promotion of seedling growth in some agricultural crops.

\section{2,4-PDCA extends the display time of cut spray-type carnation flowers by promoting flower opening as well as retarding senescence}

\subsection{Procedures for observation of flower opening and senescence}

A carnation cultivar, Dianthus caryophyllus L. "Light Pink Barbara (LPB)," which belongs to the spray type of carnation flowers having multiple flowers (buds) on a stem, was used. Flowers were harvested when the first floret out of six to eight flower buds on a stalk was partially open. Stems of cut flowers were trimmed to $60 \mathrm{~cm}$. Bunches of 5 stems, each having 5 flower buds ( 25 buds in total per bunch), were put in 900-ml glass jars containing $300 \mathrm{ml}$ of test solutions. The test solutions were distilled water (control) and the solutions containing each of PDCA analogs at given concentrations. The flowers were kept under continuous light at $23^{\circ} \mathrm{C}$ and $50-70 \%$ relative humidity. Fully open non-senescent (FONS) flowers, which were regarded as flowers ranging from Open stage 6 to Senescence stage 2 [12, 13], were counted daily, and the percentage of these flowers to the total number (25) of initial flower buds per bunch was calculated. The display time of the cut flowers in days is expressed by the number of days during which the percentage of FONS flowers was $40 \%$ or more [14, 15]. Data are 
presented as changes of the percentages of FONS flowers during 24 days. The time to flower opening was defined as the number of days from the start of experiment to the time when the percentage of FONS flowers reached $40 \%$ [16].

\subsection{Analysis of flower opening as well as display time (vase life) in cut "LPB" carnation flowers}

Figure 1 shows changes in the percentage of FONS flowers for cut "LPB" carnation flowers treated with different concentrations of 2,4-PDCA [16]. The treatment with 2,4-PDCA tended to shorten the time to flower opening, which was 4.4 days for the control, 4.3 days for $0.3 \mathrm{mM}$, 3.3 days for $1 \mathrm{mM}$, and 3.8 days for $2 \mathrm{mM}$, although it was significantly different from the control only with treatment at $1 \mathrm{mM} 2,4$-PDCA by Steel's multiple range test $(P<0.05)$. These observations suggested that 2,4-PDCA has an activity to accelerate flower bud opening. The display time was significantly lengthened by treatment with 2,4-PDCA, attaining 53, 111, and $135 \%$ increases at $0.3,1$, and $2 \mathrm{mM}$ 2,4-PDCA, respectively, as compared with the control [15].

This figure was adapted from Figure 2 in Sugiyama and Satoh [16]. The percentage of fully open and non-senescent (FONS) flowers was calculated from the proportion of those flowers to the total number of initial flower buds ( 25 buds per 5 flowers). The time to flower opening (I) is the time in days from the start of experiment until the percentage of open flowers reached at $40 \%$. The display time of the flowers (II) is the duration when the percentage of FONS flowers was $40 \%$ or more.

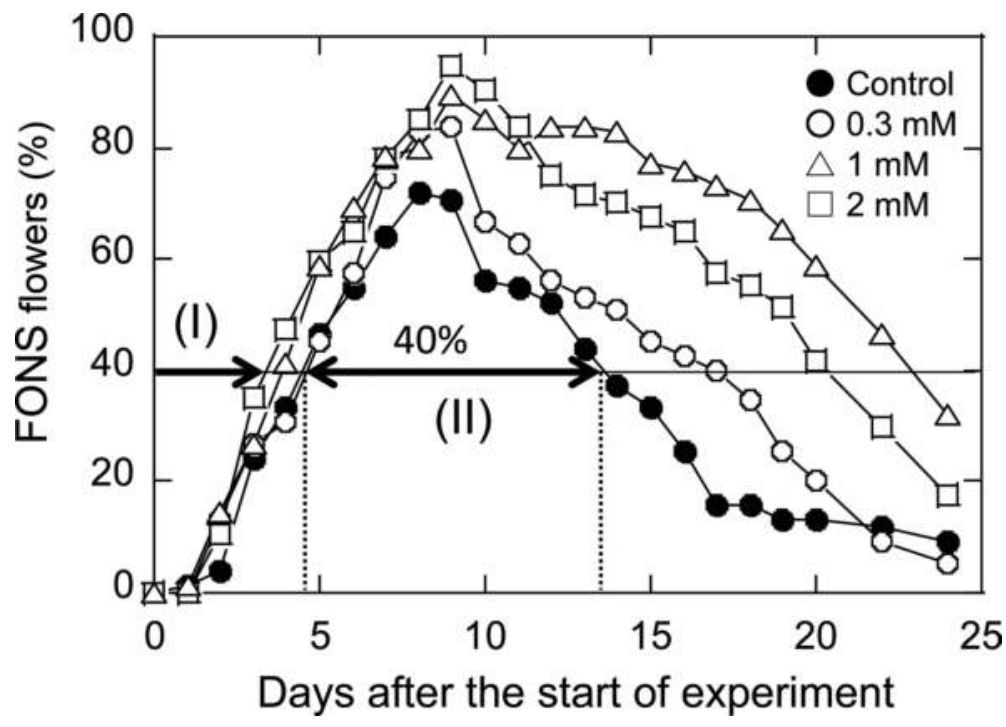

Figure 1. Profiles of flower opening and duration of display time in cut flowers of 'Light Pink Barbara' (LPB) carnation treated continuously with different concentrations of 2,4-PDCA. This figure was adapted from Figure 2 in Sugiyama and Satoh [16]. The percentage of fully-open and non-senescent (FONS) flowers was calculated from the proportion of those flowers to the total number of initial flower buds ( 25 buds per 5 flowers). The time to flower opening (I) is the time in days from the start of experiment until the percentage of open flowers reached $40 \%$. The display time of the flowers (II) is the duration when the percentage of FONS flowers was $40 \%$ or more. 


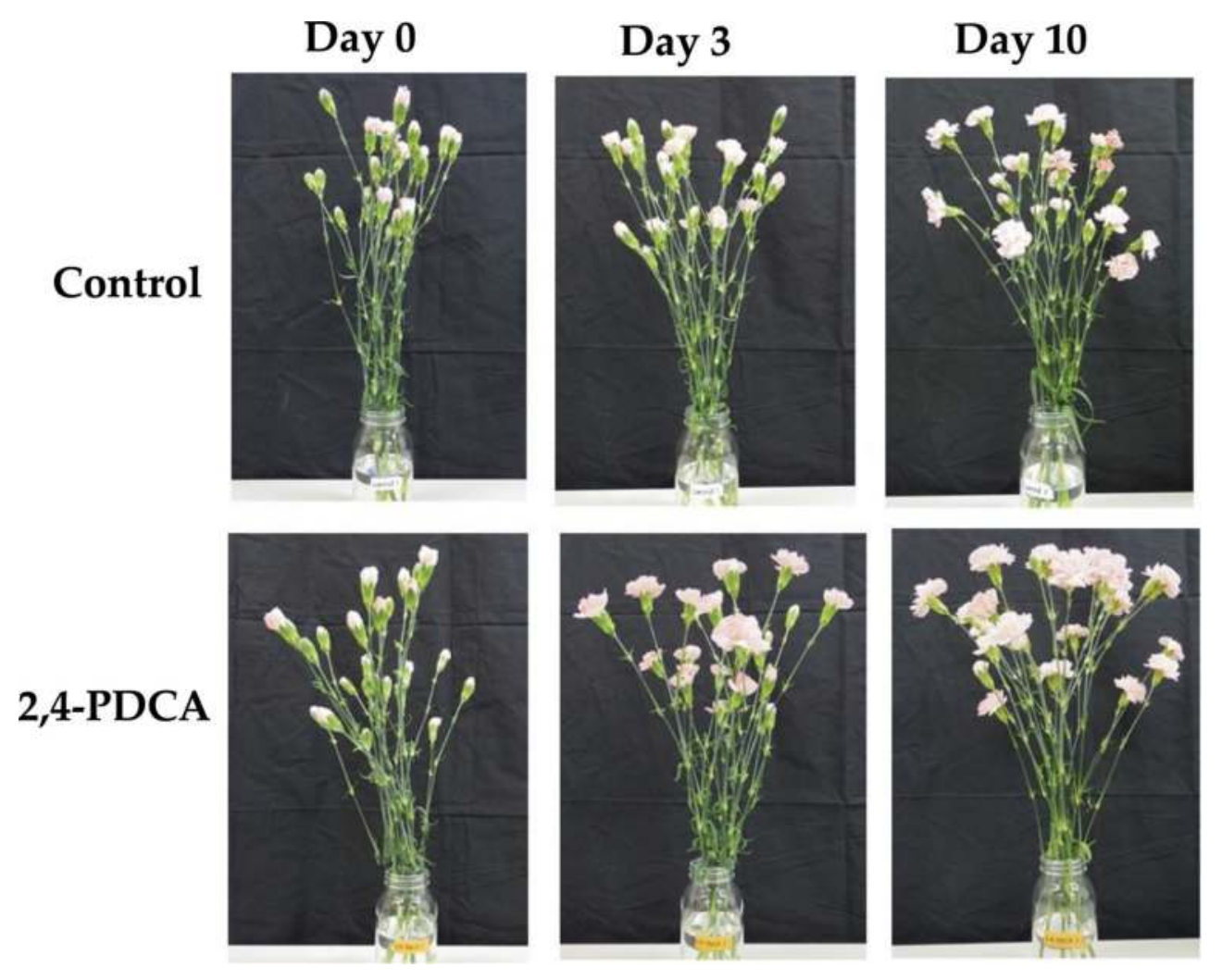

Figure 2. Flower opening and senescing profiles of cut "LPB" carnation flowers treated without (control) or with 2,4PDCA at $2 \mathrm{mM}$. Typical profiles for each treatment out of three replicates were chosen for 0,3 , and 10 days of the experiment. This figure was adapted from Figure 4 in Sugiyama and Satoh [16], by extracting only the data for 2,4-PDCA.

\subsection{Comparison of the effect of 2,4-PDCA between the continuous and pulse treatments}

In the experiment described earlier, 2,4-PDCA was applied to cut carnation flowers continuously during experiments (the continuous treatment). This procedure sometimes caused detrimental side effects, resulting in the browning of leaves or broken stalks [16], probably because of excess absorption of the chemicals. Also, this procedure seems to be practically inadequate from the application perspective of the chemicals, since it would need much labor work. Therefore, we tried to apply PDCA by a pulse treatment, in which the flowers were treated once after harvest for a short period when kept in water [17]. We treated cut "LPB" flowers with 2,4-PDCA at 0 (control), 5, or $10 \mathrm{mM}$ for $24 \mathrm{~h}$, and thereafter left with their stalk end in water (pulse treatment). The time to flower opening was $6.0 \pm 0.5$ days (shown by the mean \pm SE of three replicates) in the control. The pulse treatment with 5 and $10 \mathrm{mM} \mathrm{2,4-}$ PDCA shortened it to $5.0 \pm 0.7$ days and $3.1 \pm 0.4$ days, respectively, whereas the continuous 
treatment with $2 \mathrm{mM}$ 2,4-PDCA shortened the time to flower opening to $4.3 \pm 0.3$ days. The display time in the control was $5.7 \pm 1.7$ days. It was lengthened by the pulse treatment with 2,4-PDCA at $5 \mathrm{mM}$ to $11.9 \pm 0.7$ days and with $10 \mathrm{mM}$ to $14.2 \pm 0.3$ days, and by the continuous treatment with $2 \mathrm{mM}$ 2,4-PDCA to $11.2 \pm 0.5$ days. These results revealed that the effect of pulse treatment with 5 or $10 \mathrm{mM}$ 2,4-PDCA on the flower opening characteristics was similar to or greater than that of the continuous treatment with $2 \mathrm{mM}$ 2,4-PDCA in "LPB" carnation.

\section{PDCA analogs extend the display time of carnation flowers}

As described earlier, 2,4-PDCA stimulated flower opening and prolonged the display time in cut spray-type carnation flowers. Then, we compared 2,4-PDCA's action with those of its structural analogs (2,3-, 2,5-, 2,6-, 3,4-, and 3,5-PDCA) in order to obtain further information on 2,4-PDCA's action [16] . In this experiment, cut "LPB" carnation flowers were treated without (control) or with each of PDCA analogs at $2 \mathrm{mM}$.

Figure 2 shows flower opening and senescing profiles of the control and 2,4-PDCA-treated flowers, which were chosen as typical specimens, 0,3 , and 10 days after the start of the experiment. On day 3, open pink flowers were seen more among the flowers treated with 2,4-PDCA than in the control. These observations indicated that treatment with 2,4-PDCA accelerated flower opening as compared with the untreated control flowers. On day 10, the control flowers remained as a mixture of buds, open flowers, and senesced flowers. The senesced flowers showed in-rolling and wilting of petals, typical symptoms of senescence in response to

\begin{tabular}{ccc}
\hline Chemicals & $\begin{array}{c}\text { Time to flower } \\
\text { opening (days) }\end{array}$ & $\begin{array}{c}\text { Display time } \\
\text { (days) }\end{array}$ \\
\hline Control & $9.0 \pm 1.0$ & $8.3 \pm 1.9$ \\
2,3-PDCA & $4.0 *^{*} 0.0$ & $14.7 *^{*} 0.3$ \\
2,4-PDCA & $4.0 *^{*} 0.6$ & $14.7 *^{*} 1.7$ \\
2,5-PDCA & $4.7 *^{*} 0.3$ & $13.0 \pm 2.1$ \\
2,6-PDCA & $5.3 *^{*} 0.3$ & $9.3 \pm 1.5$ \\
3,4-PDCA & $5.7 *^{*} 0.3$ & $14.7 *_{ \pm} .4$ \\
3,5-PDCA & $4.7 *^{*} 0.7$ & $15.5 *^{*} 1.2$ \\
\hline
\end{tabular}

Table 1. Effects of PDCA analogs on the time to flower opening and the display time of cut spray "LPB" carnation flowers. The data were adapted from Table 1 in Sugiyama and Satoh [16], by extracting only the data for 2 mM PDCA. Data are shown in days with the mean \pm SE of three replicates, each with five flower stems with five florets (buds) on a stem.* shows a significant difference from the control in each column by Dunnett's multiple range test $(\mathrm{P}<0.05)$. 
ethylene. On the other hand, almost all the flowers treated with 2,4-PDCA were fully open with non-wilted and turgid petals. At the later stage, 2,4-PDCA-treated flowers withered with browning at the petal margins, as well as with jumbled, but turgid, fading petals [15].

Table 1 summarizes the effects of all the PDCA analogs on the time to flower opening and the display time of cut "LPB" flowers. The time to flower opening was 9.0 days for the control flowers and was significantly shortened by treatment with all of the PDCA analogs. It was the shortest by treatment with 2,3-PDCA and 2,4-PDCA (both 4.0 days), followed by 2,5-PDCA and 3,5-PDCA (both 4.7 days) and 2,6-PDCA (5.3 days) and 3,4-PDCA (5.7 days). Four of the PDCA analogs (2,3-, 2,4-, 3,4-, and 3,5-PDCA) significantly lengthened the display time compared with that of the control (8.3 days); the display times varied from 14.7 to 15.5 days. 2,5-PDCA and 2,6-PDCA tended to lengthen the display time, although their effects were not statistically significant.

\section{PDCA and PCA analogs stimulate root and shoot growth in lettuce, carrot, and rice seedlings}

\subsection{Effects of 2,3-PDCA and 2,4-PDCA on root elongation of lettuce, carrot, and rice seedlings}

The action mechanism of PDCAs for accelerating flower bud opening in carnation remains unresolved. Satoh et al. [15] hypothesized on the association of gibberellin (GA) with the promoting action of 2,4-PDCA on flower bud opening in cut flowers of spray-type carnation. This hypothesis arose from the notion that 2,4-PDCA is a structural analog of OxoGA, which is a cosubstrate for enzymes acting in GA biosynthesis and inactivation. We tried to test whether 2,3-PDCA and 2,4-PDCA have GA-like activity using a bioassay system, in which exogenously applied GA promotes hypocotyl elongation of lettuce seedlings [18].

Uniformly germinated seeds were placed on solidified Gellan Gum $(1.0 \%$, w/v) in a glass test tube. The Gellan gel contained 2,3-PDCA or 2,4-PDCA at 0 (control), 0.3, 1, and $3 \mathrm{mM}$, and $\mathrm{GA}_{3}$ at $0.3 \mathrm{mM}$. After 7 days at $23^{\circ} \mathrm{C}$ in the light, 2,3-PDCA at $0.1 \mathrm{mM}$ promoted root elongation, and the degree of promotion increased up to $1 \mathrm{mM}$, then declined slightly at $3 \mathrm{mM}$, whereas, 2,4-PDCA at $0.1-3 \mathrm{mM}$ severely inhibited the elongation of lettuce roots (Figure 3). $\mathrm{GA}_{3}$ at $0.3 \mathrm{mM}$ promoted hypocotyl elongation but inhibited root elongation in lettuce seedlings. Moreover, we found that 2,3-PDCA and 2,4-PDCA had similar effects on root elongation of carrot seedlings; 2,3-PDCA at $0.3-3 \mathrm{mM}$ promoted the elongation of roots, whereas 2,4-PDCA at 0.1-3 $\mathrm{mM}$ inhibited it.

\subsection{Effects of PDCA and PCA analogs on root and shoot elongation of rice seedlings}

Similar to the investigation with lettuce and carrot seedlings, the effects of PDCA analogs on root growth of rice seedlings were examined. 2,3-, 3,4-, and 3,5-PDCA promoted the root elongation, although the latter two were less effective, whereas 2,4-PDCA and 2,6-PDCA inhibited the root elongation, and the effect of 2,5-PDCA on root elongation was very small. These 


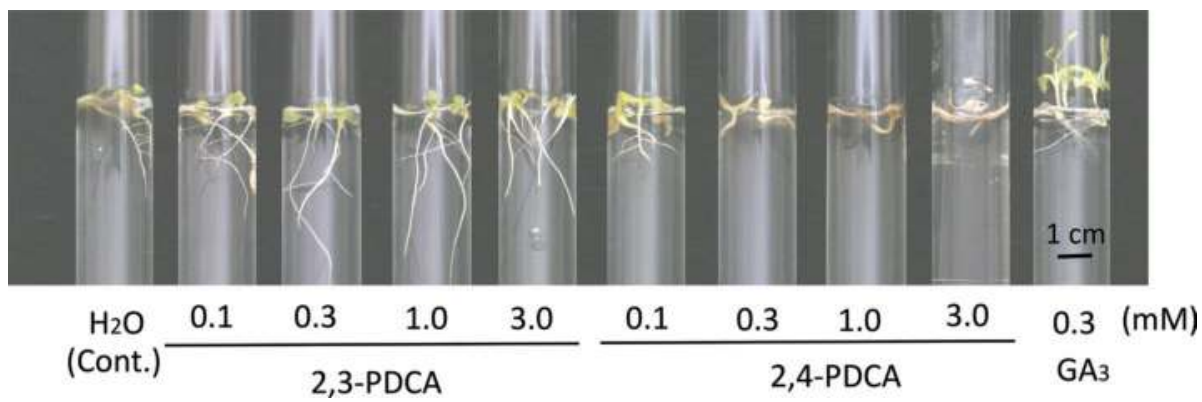

Figure 3. Effects of 2,3-PDCA, 2,4-PDCA, and $\mathrm{GA}_{3}$ in root elongation of lettuce seedlings. This figure was cited from Figure 1 in Satoh and Nomura [18]. Three germinated lettuce seeds with radicles protruded $1 \mathrm{~mm}$ were placed on the surface of solidified Gellan Gum $\left(0.1 \%\right.$, w/v) containing 2,3-PDCA and 2,4-PDCA at $0.1-3 \mathrm{mM}$ and $\mathrm{GA}_{3}$ at $0.3 \mathrm{mM}$ and allowed to grow for 7 days at $23^{\circ} \mathrm{C}$ under light from white fluorescent lamps. Water was used as the control.

results suggested that the carboxyl group at position 3 of the pyridine ring is necessary to promote root elongation in rice seedlings and probably in lettuce and carrot seedlings. Therefore, we explored the activity of PCA analogs, that is, 2-, 3-, and 4-PCA and 3-PCA amide, as well as 2,3-, 2,4-, and 3,4-PDCA, on the growth of rice seedlings grown for 7 days by hydroponic culture [18].

Uniformly germinated rice seeds were hydroponically grown in water (control) and test solutions containing PDCA or PCA analogs (2,3-, 2,4-, and 3,4-PDCA; 2-, 3-, and 4-PCAs; 3-PCA amide) at $0.03,0.1$, and $0.3 \mathrm{mM}$. Twenty-five germinated seeds were floated on a plastic mesh using a polyurethane float in a transparent plastic box containing $300 \mathrm{ml}$ of test solutions. After 7 days, the whole seedlings, and roots and shoots were photographed. The root and shoot (leaf + leaf sheath) lengths were measured with a curvemeter (Pen-type Map-meter Concurve 10, Koizumi Sokki Mfg. Co., Ltd., Nagaoka, Japan) on printed photographs. The total root length of each seedling was obtained as the sum of the lengths of a seminal root and all coronal roots. The seedlings were aligned according to the total root length, then 15 seedlings in the middle were chosen for measuring the root length. Also, the shoot length was determined similarly.

Figure 4 shows the seedlings treated with the PDCAs and PCAs at $0.3 \mathrm{mM}$. The apparent mass of roots (both seminal and coronal roots) was clearly increased by 3-PCA, 3,4-PDCA, and 2,3-PDCA, but decreased by 2-PCA, 4-PCA, and 2,4-PDCA. Interestingly, 3-PCA amide decreased the mass of roots, although not as much as 2-PCA, 4-PCA, and 2,4-PDCA. On the other hand, all the chemicals, except 4-PCA, appeared to promote the elongation of shoots, which was judged from the protrusion of shoot over the edge of containers.

Figure 5A and B show the total length of root and shoot per seedling, respectively, treated with PCA and PDCA analogs at $0.03-0.3 \mathrm{mM}$. Root elongation was significantly promoted by 3-PCA, 3,4-PDCA, and 2,3-PDCA at all the concentrations used. The magnitude of promotion was similar in 3-PCA and 3,4-PDCA, followed by 2,3-PDCA. By contrast, 2-PCA, 4-PCA, 2,4PDCA, and 3-PCA amide significantly inhibited the root elongation at all the concentrations 


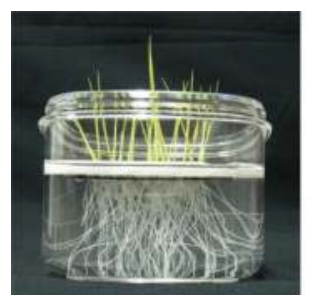

Control ( $\left.\mathrm{H}_{2} \mathrm{O}\right)$

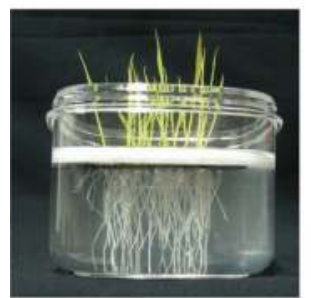

3-PCA amide

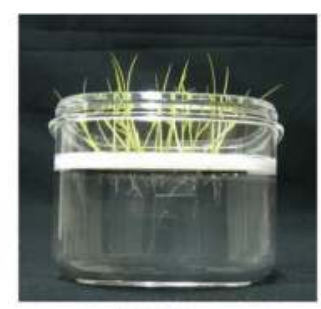

2-PCA

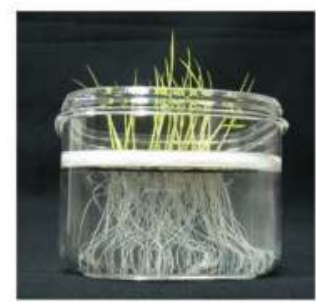

2,3-PDCA

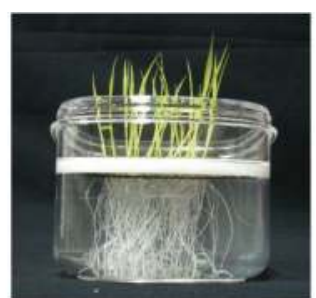

3-PCA

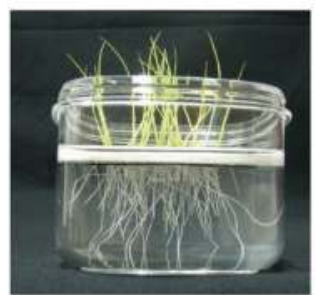

2,4-PDCA

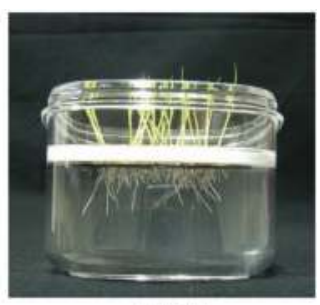

4-PCA

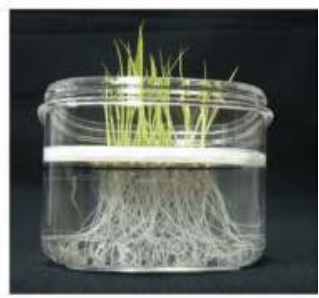

3,4-PDCA

Figure 4. Effects of PCA analogs (2-, 3-, 4-PCA, and 3-PCA amide) and PDCA analogs (2,3-, 2,4-, and 3,4-PDCA) on the growth of rice seedlings. Germinated rice seeds were grown at $23^{\circ} \mathrm{C}$ for 7 days hydroponically in test solutions containing each chemical at $0.3 \mathrm{mM}$. This figure was previously shown as Figure 5 in Satoh and Nomura [18].

A.

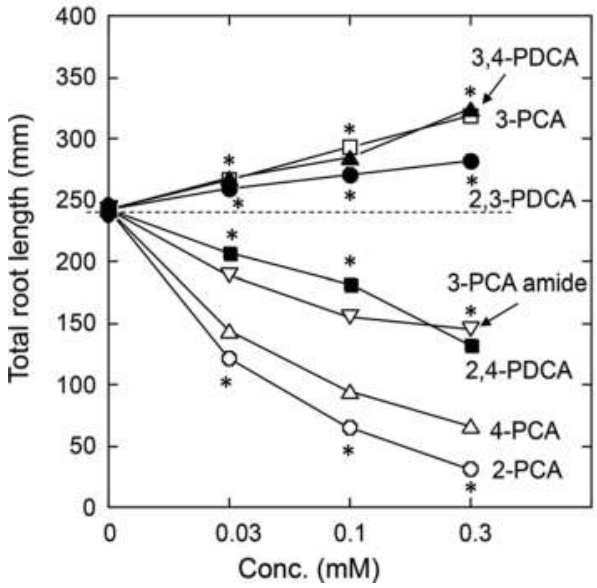

B.

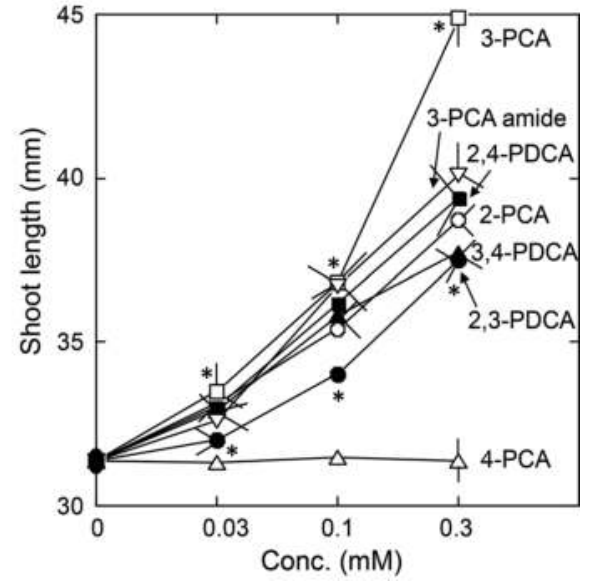

Figure 5. Effects of PCA analogs (2-, 3-, 4-PCA, and 3-PCA amide) and PDCA analogs (2,3-, 2,4-, and 3,4-PDCA) on the root elongation (A) and shoot elongation (B) of rice seedlings. * shows significant difference from the control by Dunnett's multiple range test $(P<0.05)$, and symbols between top and bottom stars at given concentrations are all significantly different from the control. This figure was previously shown as Figure 6 in Satoh and Nomura [18].

used. 2-PCA had the greatest inhibitory effect on root elongation, followed by 4-PCA, 2,4PDCA, and 3-PCA amide. On the other hand, all the chemicals, except 4-PCA, significantly promoted the elongation of shoots of rice seedlings. 3-PCA was most active in the promotion 
of shoot elongation in rice seedlings, followed by 3-PCA amide, 2,4-PDCA, and 2-PCA. 4-PCA did not affect the shoot elongation of rice seedlings.

Precise analyses of the effects of PDCA and PCA analogs revealed that the acceleration of root elongation in rice seedlings depends on the free carboxyl group $(-\mathrm{COOH})$ at position 3 of the pyridine ring, and the inhibition of root elongation depends on $-\mathrm{COOH}$ at positions 2 or 4 of the ring (Figures 3-6). When the second carboxyl group was introduced into 3-PCA, its introduction to position 2 of the pyridine ring, which makes 2,3-PDCA, reduced the promoting activity of 3-PCA more severely than that into position 4 of the pyridine ring, which makes 3,4-PDCA (Figures 4 and 5). Moreover, in PDCAs, 3-COOH overcame the inhibitory effects of 2- or 4-COOH; in other words, the latter two -COOHs could not nullify the promotive effect of 3-COOH. Interestingly, when 3-COOH of 3-PCA was replaced with $-\mathrm{CONH}_{2}$, the resultant 3-PCA amide lost the root elongation promoting activity in rice seedlings (Figure 5). Both 3-PCA and 3-PCA amide are vitamin B3, known as nicotinic acid and nicotinamide, respectively, and are regarded to have an activity equivalent to the vitamin. The present findings that 3-PCA promoted but 3-PCA amide inhibited rice root elongation suggested that the root elongation promoting the activity of 3-PCA did not originate from its activity as vitamin B3. The present findings showed the promoting effects of 3-PCA and PDCA analogs with $3-\mathrm{COOH}$ on root elongation in rice seedlings. This notion may also apply to the promotion of root elongation in lettuce and carrot seedlings.

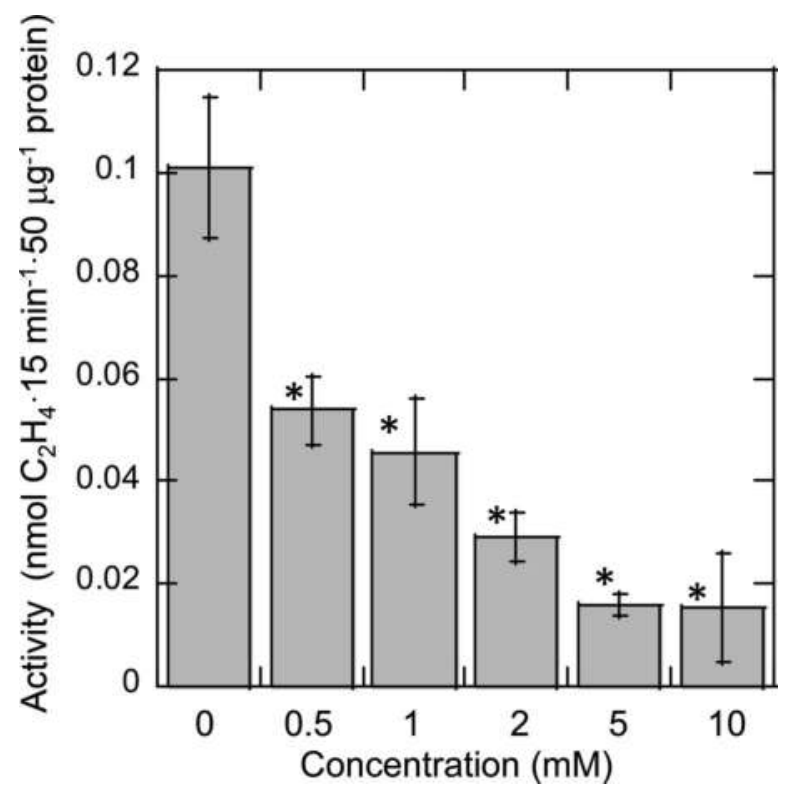

Figure 6. The inhibition of ACC oxidase activity by 2,4-PDCA. ACC oxidase was prepared by the expression of carnation DcACO1 cDNA in E. coli, and its activity was determined at $1 \mathrm{mM} \mathrm{ACC}$ in the absence or presence of 2,4-PDCA at the given concentrations. Data are the means \pm SE of triplicate determinations. This figure was adapted from Figure 1 in Satoh et al. [15]. 


\section{Search for a possible biochemical action mechanism of PDCA and PCA analogs}

We searched for possible biochemical and molecular mechanism of PDCA action. So far, we tested effects of 2,4-PDCA on recombinant ACC oxidase activity [15] and on gibberellin action by examining the changes in gene expression of DELLA protein (GAI), a negative regulator of GA signaling, and GA contents in carnation flowers [19].

First, we examined the action of 2,4-PDCA on ACC oxidase, which was synthesized in Escherichia coli cells from carnation ACC oxidase gene (DcACO1). For the construction of an expression plasmid, the entire coding region was amplified from DcACO1 cDNA [20] by PCR. DcACO1 protein was expressed from DcACO1 cDNA in E. coli cells as described elsewhere [21]. ACC oxidase activity was assayed by the standard method [21]. The recombinant ACC oxidase had a Km of $118 \mathrm{mM}$ for ACC. This $\mathrm{Km}$ value was comparable to that reported previously for ACC oxidase from carnation petals, $30-425 \mathrm{mM}$ depending on $\mathrm{CO}_{2}$ concentration [22] and 111-125 $\mathrm{mM}$ in the presence of $\mathrm{NaHCO}_{3}$ [23].

Figure 6 shows the inhibitory effects of 2,4-PDCA on the activity of the recombinant ACC oxidase. 2,4-PDCA inhibited an enzyme activity by $47 \%$ at $0.5 \mathrm{mM}$, and the magnitude of inhibition increased gradually as its concentration increased, attaining $85 \%$ inhibition at $10 \mathrm{mM}$. The results confirmed that 2,4-PDCA inhibited the activity of ACC oxidase. Iturriagagoittia-Bueno et al. [10] showed that OxoGA inhibited ACC oxidase by competing with ascorbate. 2,4-PDCA was shown to compete with OxoGA in the inhibition of vertebrate collagen proline-4-hydroxylase, an OxoGA-dependent dioxygenase [2, 3].

Therefore, it is speculated that 2,4-PDCA inhibits ACC oxidase activity by competing with ascorbate, although there is a possibility that 2,4-PDCA inhibited ACC oxidase activity by chelating $\mathrm{Fe}^{2+}$ [24].

Next, we examined the expression profiles of DcGAI gene in carnation flowers by qRT-PCR analysis. DcGAI was expressed in all the tissues examined, that is, calyx, style, receptacle, ovary, and petals. A high level of expression was observed in calyx, and the expression levels in style, receptacle, ovary, and petals were low. We also analyzed the changes in expression levels of DcGAI in petals of carnation flowers during the flower opening process. The transcript level of DcGAI was high at the early stages of flower opening and decreased at later stages of flower opening. We examined the expression of the gene in 2,4-PDCA-treated flowers. Flowers at Opening stage (Os) 1 to Os 2 were treated with $2 \mathrm{mM}$ 2,4-PDCA for 10 days. Using petals sampled from these flowers, the expression of DcGAI was examined by qRTPCR. The expression of the gene was decreased in accordance with the progression of the flower opening process in the control flowers. By contrast, the expression of DcGAI was maintained at a high level in 2,4-PDCA-treated flowers and was significantly higher than that in the control flowers at day 10 .

Thus, an increase in the transcript level of DcGAI was observed in 2,4-PDCA-treated flowers. GAI is a negative regulator of GA action and suppresses growth and elongation [25]. Therefore, it is supposed that the increase in DcGAI expression leads to the suppression of petal 
cell growth, which does not coincide with the enhancement of flower opening. Therefore, it is likely that the alteration of GAI expression is not related to the enhancing effect of 2,4-PDCA.

Furthermore, we examined GA contents as affected by 2,4-PDCA treatment in opening carnation flowers by LC-MS/MS analysis. Although endogenous GAs acting in carnation have not been identified so far, we set out to measure the GA 1 content since several ornamental plants (Chrysanthemum morifolium, Eustoma grandiflorum, Gentiana triflora, Phalaenopsis hybrid, and petunia) contain $\mathrm{GA}_{1}$ as a bioactive GA [26]. However, $\mathrm{GA}_{1}$ was not detected in carnation petals in our LC-MS/MS analysis. We found $\mathrm{GA}_{3}$ accumulated instead and determined the $\mathrm{GA}_{3}$ content in petals of opening flowers. In this experiment, all the flowers starting from Os 2 reached Os 4-6 in 4 days in the control flowers. 2,4-PDCA treatment accelerated flower opening, and all the treated flowers reached Os 4-6 in 2 days. We measured GA content in $_{3}$ the non-treated flowers at days $0-4$ and found that the $\mathrm{GA}_{3}$ level tended to be decreased in the course of flower opening. We also measured $\mathrm{GA}_{3}$ content in 2,4-PDCA-treated flowers at day 1 , when the treated flowers showed a significant increase in the number of open flowers. We observed a tendency that $\mathrm{GA}_{3}$ content in the 2,4-PDCA-treated flowers was lower than that in the control. The $\mathrm{GA}_{3}$ content in the control flowers was $48.5 \pm 10.0 \mathrm{pmol} \cdot \mathrm{g}^{-1} \mathrm{FW}$, whereas it was $26.6 \pm 14.3 \mathrm{pmol} \cdot \mathrm{g}^{-1} \mathrm{FW}$ in the 2,4-PDCA-treated flowers. There was no significant difference between the control and the treated samples by $t$-test at $P<0.05$. These results showed that 2,4-PDCA increases the gene expression of the growth suppressor, GAI, and decreases the GA level, suggesting that GA signaling and action are altered by 2,4-PDCA treatment. However, such changes are contradictory to the enhancement of flower opening, which suggests that GA is not associated with the enhancing effect of 2,4-PDCA in carnation flowers.

\section{Discussion}

Our studies showed that PDCAs accelerate flower opening and retard senescence, which increase the number of open flowers, resulting in the extension of the display time of cut flowers of "LPB" carnation. At first, this finding was obtained by the experiment with 2,4-PDCA and then subsequent experiments with other PDCA analogs, that is, 2,3-, 2,5-, 2,6-, 3,4-, and 3,5-PDCA. Judging from their effectiveness in the acceleration of flower opening and extension of display time, 2,3PDCA and 2,4-PDCA were thought to be suitable agents for the treatment of the flowers.

2,4-PDCA is a structural analog of OxoGA and has been suggested to inhibit OxoGAdependent dioxygenases by competing with OxoGA and ethylene production in detached carnation flowers by competing with ascorbate on ACC oxidase action [4, 11]. We confirmed that 2,4-PDCA inhibited ACC oxidase action with a recombinant enzyme produced in E. coli cells from carnation DcACO1 cDNA. These findings suggested that 2,4-PDCA primarily inhibited ACC oxidase action, resulting in the inhibition of ethylene production and the delay of withering of carnation flowers. However, probably, this mechanism of action could not be applicable for PDCA analogs other than 2,4-PDCA. Meanwhile, it was suggested that GA might be involved in the action of 2,4-PDCA on flower opening and senescence of carnation flowers [15]. However, 2,4-PDCA treatment gave no significant effects on the expression of DcGAI gene, which is a key factor in the GA-signaling pathway and on GA content in the flower petals 
[19]. Therefore, at present, there is no reliable explanation for the PDCAs' action mechanism in the stimulation of flower opening and extension of display time in carnation flowers.

It was revealed that 2,3-PDCA promoted root elongation in lettuce, carrot, and rice seedlings, whereas 2,4-PDCA inhibited it [18]. The action of PDCA and PCA analogs on root and shoot elongation was further examined using rice seedlings. 2,3-, 3,4-, and 3,5-PDCA promoted rice root elongation, whereas 2,4- and 2,6-PDCA inhibited it, and 2,5-PDCA had little effect. 3-PCA (nicotinic acid) promoted rice root elongation, but 2- and 4-PCA did not. Moreover, 3-PCA amide (nicotinamide) did not promote root elongation. These findings indicated that a carboxyl group substituted on position 3 of the pyridine ring is necessary to promote root elongation, and that the promoting effect of 3-PCA was not from its action as vitamin B3, but from its intrinsic activity as a 3-COOH-substituted pyridine. On the other hand, all the PCA and PDCA analogs tested in this study, except for 4-PCA, promoted shoot elongation of rice seedlings. The mechanism of action of PCA and PDCA analogs on root growth will hopefully be elucidated in the near future.

We have observed that PDCAs are practically applicable to cut flowers of spray-type carnation cultivars other than "LPB." In addition, this issue will also be examined with other species of ornamentals, of which flowers are used as spray-type flowers, such as Eustoma, Gypsophila, and Alstroemeria flowers and spray-type Chrysanthemum. The promotion of root elongation in the seedling of vegetable and ornamental crops by PDCA and PCA analogs probably has merits in practical agriculture, since massive roots of seedlings surely promote root establishment of the seedlings after transplanting. From this point of view, we are now investigating a practical method to apply these chemicals to grow sound and vigorous seedlings of vegetable and ornamental crops.

\section{Acknowledgements}

This study was supported financially by a Matching Planner Program Grant (MP27115663028 to S. Satoh) from the Japan Science and Technology Agency and a Grant-in-Aid (16 K07604 to S. Satoh) for Scientific Research from the Japan Society for the Promotion of Science.

\section{Conflict of interest}

The author declares no conflict of interest.

\section{Author details}

Shigeru Satoh

Address all correspondence to: ssatoh@agr.ryukoku.ac.jp

Faculty of Agriculture, Ryukoku University, Otsu, Japan 


\section{References}

[1] Murakami K, Haneda M, Hosokawa Y, Yoshino M. Prooxidant action of pyridine carboxylic acids: Transition metal-dependent generation of reactive oxygen species. Trace Nutrition Research. 2007;24:49-55. (In Japanese with English summary)

[2] Kivirikko KI, Myllyharju J. Prolyl 4-hydroxylases and their protein disulfide isomerase subunit. Matrix Biology. 1998;6:357-368

[3] Kivirikko KI, Pihlajaniemi T. Collagen hydroxylases and the protein disulfide isomerase subunit of prolyl 4-hydroxylases. Advances in Enzymology and Related Areas of Molecular Biology. 1998;72:325-398

[4] Vlad F, Tiainen P, Owen C, Spano T, Daher FB, Oualid F, Senol NO, Vlad D, Myllyharju J, Kalaitzis P. Characterization of two carnation petal prolyl 4 hydroxylases. Physiologia Plantarum. 2010;140:199-207

[5] Hedden P, Kamiya Y. Gibberellin biosynthesis: Enzymes, genes and their regulation. Annual Review of Plant Physiolology and Plant Molecular Biology. 1997;48:431-460

[6] Lange T, Hedden P, Graebe JE. Expression cloning of a gibberellin 20-oxidase, a multifunctional enzyme involved in gibberellin biosynthesis. Procedings of National Academy of Sciences USA. 1994a;91:8552-8556

[7] Lange T, Schweimer A, Ward DA, Hedden P, Graebe JE. Separation and characterization of three 2-oxoglutarate-dependent dioxygenases from Cucurbita maxima L. endosperm involved in gibberellin biosynthesis. Planta. 1994b;195:98-107

[8] Smith VA, MacMillan J. Purification and partial characterization of a gibberellin 2 $\beta$-hydroxylase from Phaseolus vulgaris. Journal of Plant Growth Regulation. 1984;2:251-264

[9] Smith VA, MacMillan J. The partial purification and characterisation of gibberellin 2 $\beta$-hydroxylases from seeds of Pisum sativum. Planta. 1986;167:9-19

[10] Iturriagagoittia-Bueno T, Gibson EJ, Schofield CJ, John P. Inhibition of 1-aminocyclopropane-1-carboxylate oxidase by 2-oxoacid. Phytochemistry. 1996;43:343-349

[11] Fragkostefanakis S, Kalaitzis P, Siomos AS, Gerasopoulos D. Pyridine 2,4-dicarboxylate downregulated ethylene production in response to mechanical wounding in excised mature green tomato pericarp discs. Journal of Plant Growth Regulation. 2013;32:140-147

[12] Harada T, Torii Y, Morita S, Masumura T, Satoh S. Differential expression of genes identified by suppression subtractive hybridization in petals of opening carnation flowers. Journal of Experimental Botany. 2010;61:2345-2354

[13] Morita S, Torii Y, Harada T, Kawarada M, Onodera R, Satoh S. Cloning and characterization of a cDNA encoding sucrose synthase associated with flower opening through early senescence in carnation (Dianthus caryophyllus L.). Journal of Japanese Society for Horticultural Science. 2011;80:358-364 
[14] Satoh S, Nukui H, Inokuma T. A method for determining the vase life of cut spray carnation flowers. Journal of Applied Horticulture. 2005;7:8-10

[15] Satoh S, Kosugi Y, Sugiyama S, Ohira I. 2,4-Pyridinedicarboxylic acid prolongs the vase life of cut flowers of spray carnations. Journal of Japanese Society for Horticultural Science. 2014;83:72-80

[16] Sugiyama S, Satoh S. Pyridinedicarboxylic acids prolong the vase life of cut flowers of spray-type 'light pink Barbara' carnation by accelerating flower opening in addition to an already-known action of retarding senescence. Horticulture Journal. 2015;84:172-177

[17] Satoh S, Nomura Y, Morita S, Sugiyama S. Further characterization of the action of pyridinedicarboxylic acids: Multifunctional flower care agents for cut flowers of spray-type carnation. Journal of Applied Horticulture. 2016;18:3-6

[18] Satoh S, Nomura Y. Promotion of root elongation by pyridinecarboxylic acids known as novel cut flower care agents. Plant Root. 2017;11:40-47

[19] Morita S, Sugiyama S, Nomura Y, Masumura T, Satoh S. Gibberellin is not associated with the enhancing effect of 2,4-pyridinedicarboxylic acid on flower opening of 'Light Pink Barbara' carnation. Horticulture Journal. 2017;86:519-527

[20] Kosugi Y, Shibuya K, Tsuruno N, Iwazaki Y, Mochizuki A, Yoshioka T, Hashiba T, Satoh $\mathrm{S}$. Expression of genes responsible for ethylene production and wilting are differently regulated in carnation (Dianthus caryophyllus L.) petals. Plant Science. 2000;158:139-145

[21] Satoh S, Kosugi Y. Escherichia coli-based expression and in vitro activity assay of 1-aminocyclopropane-1-carboxylate (ACC) synthase and ACC oxidase. In: Binder BM, Schaller GM, editors. Ethylene Signaling: Methods and Protocols. New York: Springer; 2017. pp. $47-58$

[22] Nijenhuis-de Vries MA, Woltering EJ, De Vrije T. Partial characterization of carnation petal 1-aminocyclopropane-l-carboxylate oxidase. Journal of Plant Physiology. 1994;144: 549-554

[23] Kosugi Y, Oyamada N, Satoh S, Yoshioka T, Onodera E, Yamada Y. Inhibition by 1-aminocyclobutane-1-carboxylate of the activity of 1-aminocyclopropane-1-carboxylate oxidase obtained from senescing petals of carnation (Dianthus caryophyllus L.) flowers. Plant \& Cell Physiology. 1997;38:312-318

[24] Smith JJ, Ververidis P, John P. Characterization of the ethylene-forming enzyme partially purified from melon. Phytochemistry. 1992;31:1485-1494

[25] Harberd NP, Belfield E, Yasumura Y. The angiosperm gibberellin-GID1-DELLA growth regulatory mechanism: How an "inhibitor of an inhibitor" enables flexible response to fluctuating environments. The Plant Cell. 2009;21:1328-1339

[26] Koshioka M. Gibberellin metabolism and its regulation in horticultural plants. Regulation of Plant Growth \& Development. 2004;39:1-9 (In Japanese) 